\title{
OPTIMUM PATH FINDING FROM MULTI - PATHS BASED ON FUZZY LOGIC SYSTEM
}

\author{
Dr. Eyad I. Abba \\ Department of Electrical Eng. \\ University of Technology \\ Baghdad, Iraq
}

\author{
Dr. Sundus D. Hasan \\ Department of Electrical Eng. \\ University of Technology \\ Baghdad, Iraq
}

\author{
Rawaa Jawad \\ Department of Electrical Eng. \\ University of Technology \\ Baghdad, Iraq
}

\begin{abstract}
This paper introduced the fuzzy logic technique to find the optimum path from multipaths. The fuzzy logic system used six input as: distance (front, left obstacle, right obstacle, angle goal, turn and distance goal) that have been membership function for each input, as well as output direction, the success of the fuzzy logic algorithm and ensure the optimum path obtained from six paths, these paths takes at different rules of fuzzy logic system . Fuzzy logic technique is not an optimization technique as the particle swarm technique and the genetic algorithm are considered optimization, but in this research get several paths after changing the rules and then the optimal path is obtained from these paths in terms of short length and minimum time of implementation. This technique give the best result as well as the sixth red path is best path which is characterized by its short length and lowest processing time, This technique applied of mobile robots and use in engineering, medical, military and industrial application.
\end{abstract}

Keywords- path finding, fuzzy logic, mobile robot, obstacles

\section{INTRODUCTION}

The robot is necessary element in society today's. Many frequently jobs, and without used human facility. The word of robots are utilize in wide range for mechanical mobility machine [1] [2]. Path planning of mobile robot, located in environment with obstacles, is define to find path robot to reach from source to destination without hitting of obstacle [3], topics as shortness of path and simple are criteria of optimality to select path. Determining path from completeness is divided two types of constraint satisfaction and accurate. The first type is find optimal path or prove there is no path and in term is time consuming of time complexity, [2], So the second type find appropriate path in short time [3]. Many researchers study a mobile robot as: Weria Khaksar et.al (2015) [4] present a review motion path of mobile robot planning in unknown environments because robotics are achieved enormous in recent years for high demand in factories and carry highprecision jobs. Mihai Duguleana et.al (2016) [5] propose Q-learning and artificial neural network for path planning in static and dynamic obstacles. The algorithms presented effective in navigation scenario that information global. Result illustrate that algorithms give good rate conversion computed at speed satisfying. Akram Adib et. al (2017) [6] in this paper proposed Autonomously navigation mobile robots in unknown environments. By design fuzzy logic to make the easier navigation and use initial knowledge of reinforcement learning for coordinating behavior that caused robot to select the best action in any situation. The result show fuzzy logic and learning automata of navigation robot is give better performance in convergence and learning speed relatively of fuzzy logic and Q-algorithm. Fiorato Nicola et.al (2018) [7] propose neural network Long Short-Term Memory (LSTM) online path planning of mobile robots in unknown environment, structure LSTM is analyzed. Then compared the result between LSTM with $A^{*}$ algorithm LSTM is give good performance. H. Bharadwaja et.al (2018) [8] present Neural Networks of mobile robot to path planning, parameters take for training time, performance of network, forecasted distance is considered after iterating for achieve optimal dataset using Probabilistic Roadmap (PRM) algorithm. Improvement $36 \%$ in forecasted distance achieve use neural network and then compared with traditional PRM algorithm

\section{PATH FINDING}

Since 1980, Many research employment to solve issues of mobile robots path planning. By applied two ways as: Firstly using global scenario, obstacle information and characteristics of robot. Second way collect local information during sensor and explain path traversing problem. Developing effective trajectory planning employ optimization technique to strike trade-off between reactive to environmental event and. [9]. Finding a path to a robot is a common problem in portable robots. the robot should be able to 
move itself from the start Location to the target location without colliding with obstacles. So an important research topic in this area is navigation of autonomous portable robots, which are to find universally optimized. The path from starting point to target in particular environment. At the same time avoid collisions. That's where the path is optimized. Means that the path must meet certain criteria such as length. The path is shorter, or the power consumption of the robot is the lowest or the time required to reach the goal is minimal etc. Many artificial intelligence techniques and algorithms have been used to find path as (Fuzzy, ANN, ANFIS, ACO, PSO, Bee colony, Genetic).

\section{ARTIFICIAL INTELLIGENCE TECHNIQUES}

Artificial Intelligence (AI) has seen tremendous progress in recent years. It is a thriving research area with an increasing number of important research and basic technology areas for an increasing number of application areas. In addition to algorithmic innovations. Artificial intelligence is being utilized to enhance sciences and technologies due to its amazing capability of dealing with big data, complexity, high accuracy, and speedy processing. Artificial Neural Network (ANN), Fuzzy Logic, Neuro - Fuzzy Interference System (ANFIS), Genetic algorithm (GA), Particle Swarm Optimization (PSO), etc. are the familiar tools of Artificial Intelligence, AI has been employed in various areas such as engineering , science, medicine, computing, finance, economics and so on.

\section{FUZZY LOGIC SYSTEM}

First proposed of fuzzy logic by Zadeh in 1965 and it's based on fuzzy sets concept. Theory of Fuzzy sets are provides mean of representing uncertainty, theory of probability is primary tool of analyzing uncertainty, and suppose that uncertainty is random process. Though, uncertainty not all is random [10], The basic problem in the robot path is the need to deal with it. With a huge amount of possibilities. The advantage fuzzy logic is its ability to use common sense to describe complex systems. Very responsive. The system is complicated in a simple way described by creating a set of input and output set Variables and generate a simple rule based matrix Using fuzzy logic. The fuzzy system is composed of the following four elements [10-13]: a rule base (a set of If - Then rules), that contain quantification fuzzy logic of expert's linguistic explanation for good performance.

An interface fuzzification, that convert input into data that mechanism inference can apply to apply rules.

An interface defuzzification, that convert conclusion of mechanism inference to actual input of process

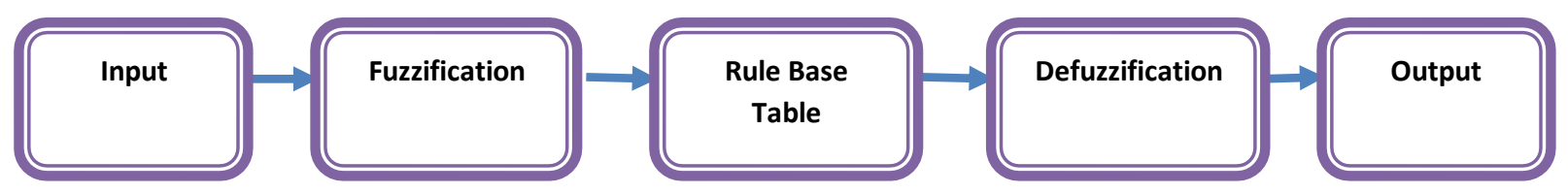

Fig1: Proposed fuzzy logic system

The path using fuzzy logic is runs of number of nodes along path. flow char of mobile robot using fuzzy logic system show in Fig (2) .In this paper, fuzzy has been utilized six input to determine the path illustrated in Fig(3) one of inputs represents distance front and has three membership function (Mfs) (low, medium and large), left obstacle has two membership function (small, medium), right obstacle has two membership function (small, medium), angle goal has five membership function (negative, no, positive, more negative, more positive), turn has two membership function (left, right), distance goal has three membership function (low, medium, and high) as well as output variable direction has five membership function (left, right, no , more left, more right) described in Fig (4). 


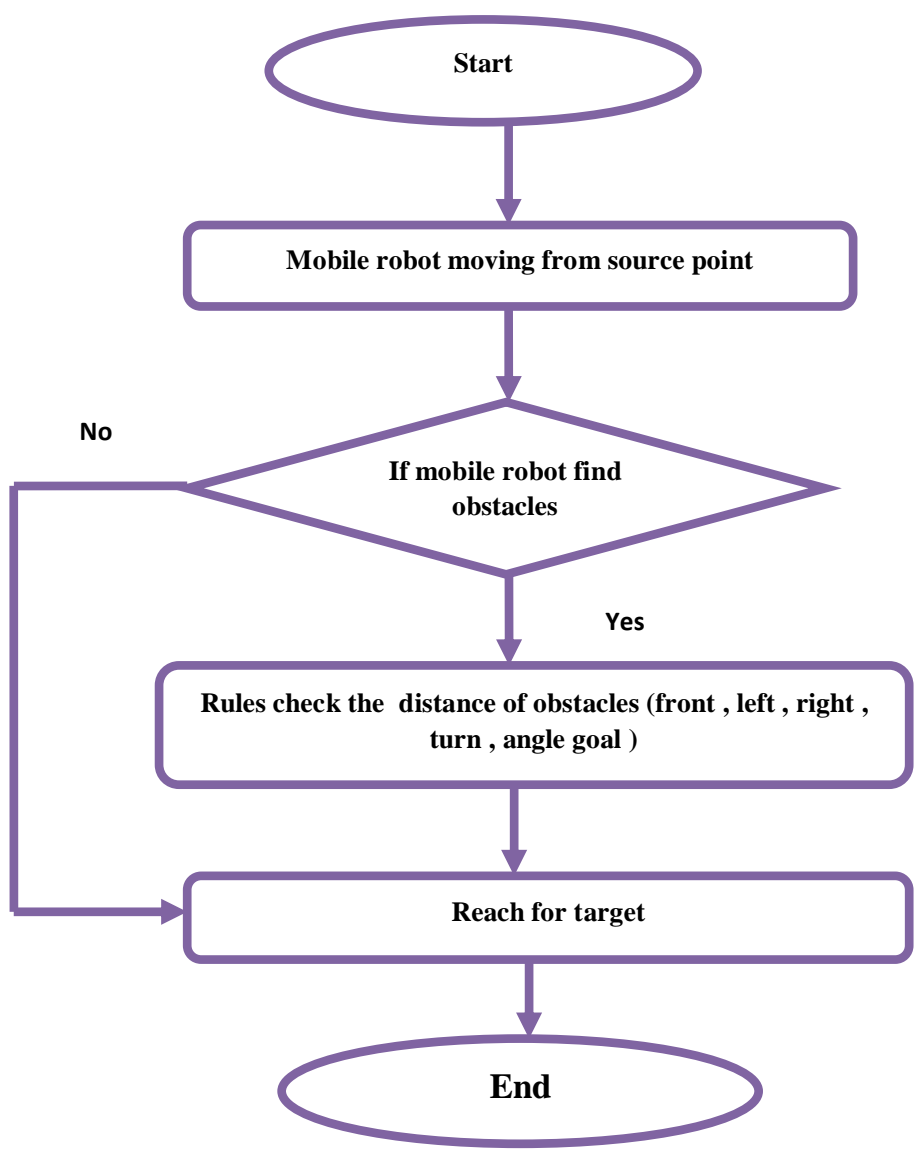

Fig 2: Flow chart for path finding of mobile robot using fuzzy logic system

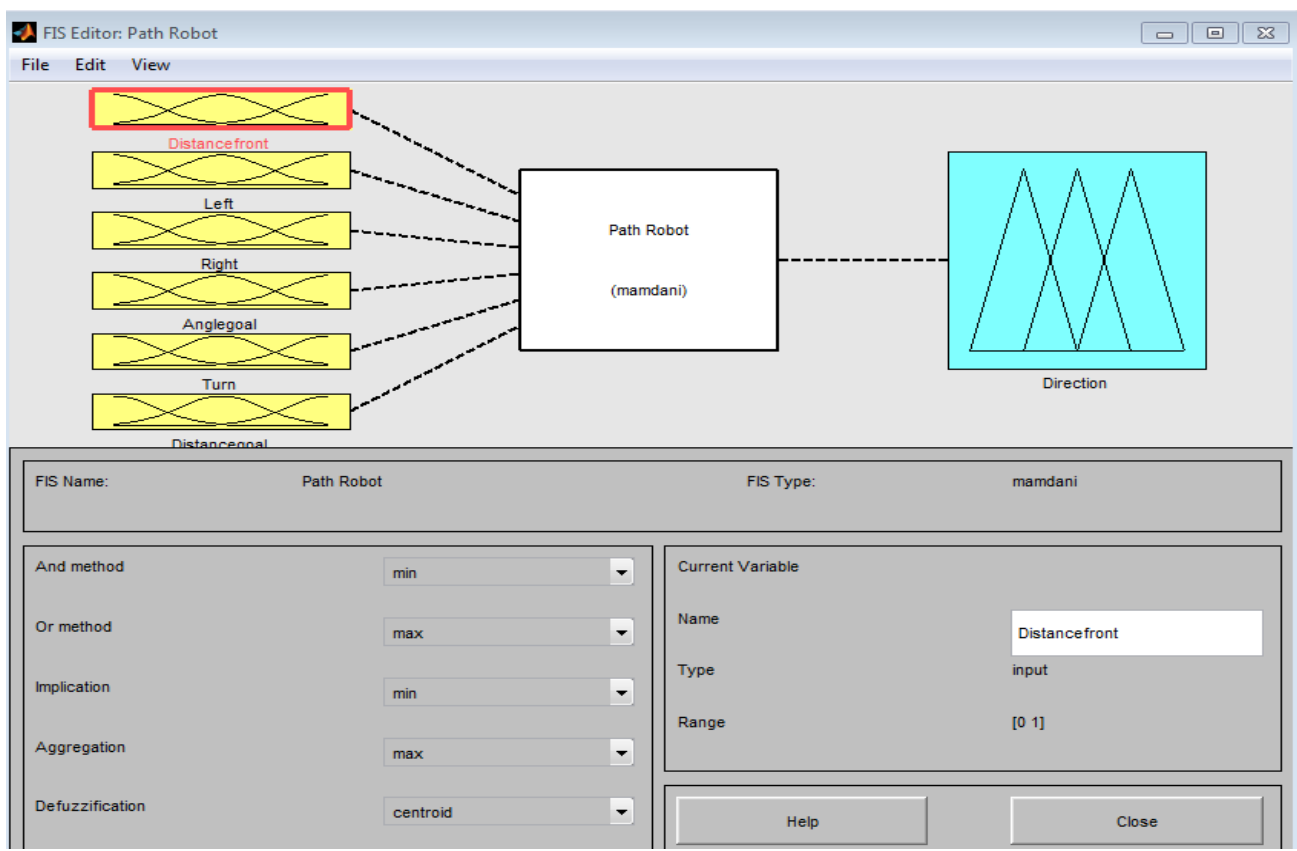

Fig (3) Inputs and output fuzzy logic system 
International Journal of Engineering Applied Sciences and Technology, 2020

Vol. 5, Issue 4, ISSN No. 2455-2143, Pages 39-46

Published Online August 2020 in IJEAST (http://www.ijeast.com)

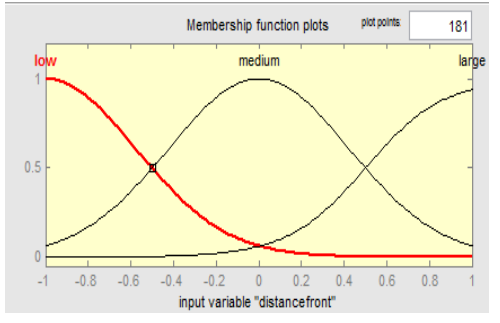

-a- MFs. distance front

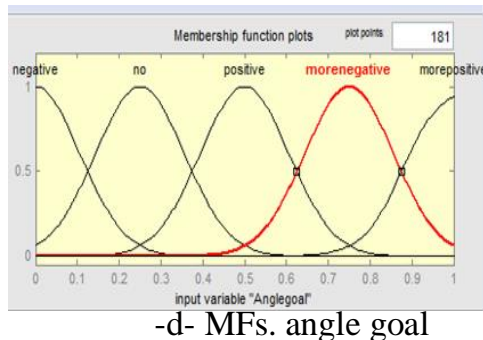

Membership function plots

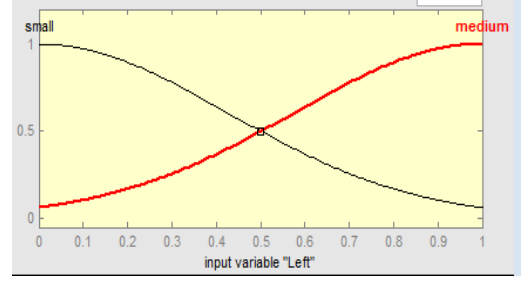

-c- MFs. distance right

-b- MFs. distance left
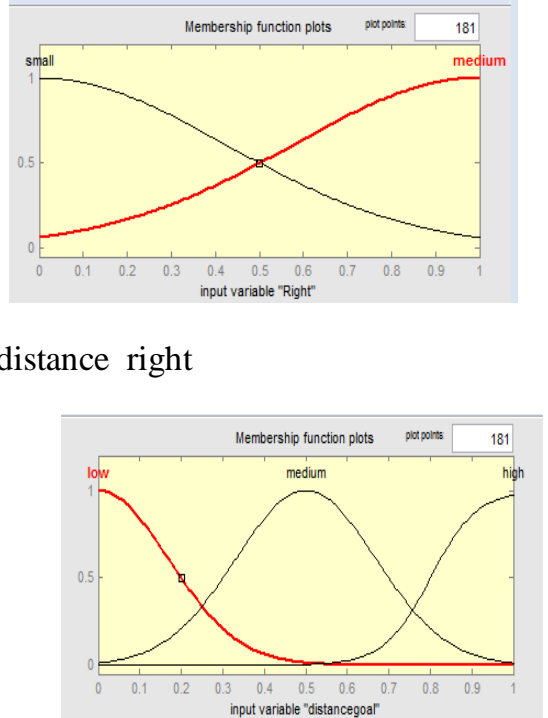

-f- MFs. distance goal

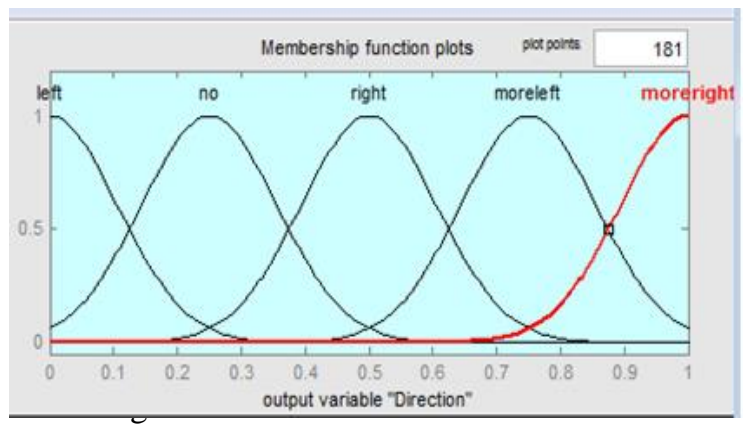

Fig (4) Membership function of inputs and outputs fuzzy logic system

\section{THE SIMULATED RESULTS}

Fuzzy logic system for path finding by using Matlab (2014a), Toolbox (Fuzzy logic system) and algorithm to inter six input as: distance (front, left, right, angle goal, turn and distance goal) that have been membership function for each input, as well as output direction, then the rules that illustrate in Table (I) applied. These save as file in Matlab with name (path robot.fis) and then call it in algorithm that built using $\mathrm{m}$-file for path finding of mobile robot. Algorithm is a function for calculating distances from obstacles, selecting source position in $\mathrm{Y}, \mathrm{X}$ format, goal position in $\mathrm{Y}, \mathrm{X}$ format, initial heading direction and safety distance $S=10$. When mobile robot moves, it continuously measures the distance from different directions. There is a comparison made between the distance and a threshold value which is equal to 30 $\mathrm{cm}$. Figs (5), (6), (7) and (8) shows the rule base editor, rule viewer, surface viewer and paths resulting from the algorithm (multiple paths in one form) where optimum path obtained from six paths, these paths takes at different rules of fuzzy logic system show in Table (II). These paths can be applied to find a path for a mobile robot.

Table (I) The fuzzy rules

\begin{tabular}{|l|l|l|l|l|l|l|l|}
\hline $\begin{array}{l}\text { Number of } \\
\text { rules }\end{array}$ & $\begin{array}{l}\text { Distance } \\
\text { front }\end{array}$ & Left & Right & Angle goal & Turn & $\begin{array}{l}\text { Distance } \\
\text { goal }\end{array}$ & Direction \\
\hline 1. & Low & None & None & None & Left & None & More left \\
\hline
\end{tabular}


International Journal of Engineering Applied Sciences and Technology, 2020

Vol. 5, Issue 4, ISSN No. 2455-2143, Pages 39-46

Published Online August 2020 in IJEAST (http://www.ijeast.com)

\begin{tabular}{|c|c|c|c|c|c|c|c|}
\hline 2. & Medium & None & None & None & Left & None & Left \\
\hline 3. & Large & None & None & None & Left & None & Left \\
\hline 4. & Low & None & None & None & Right & None & More right \\
\hline 5. & Medium & None & None & None & Right & None & Right \\
\hline 6. & Large & None & None & None & Right & None & Right \\
\hline 7. & None & Small & None & None & None & None & More right \\
\hline 8. & None & Medium & None & None & None & None & Right \\
\hline 9. & None & None & Small & None & None & None & More right \\
\hline 10. & None & None & Medium & None & None & None & Left \\
\hline 11. & None & None & None & Negative & None & Low & More left \\
\hline 12. & None & None & None & $\begin{array}{l}\text { More } \\
\text { negative }\end{array}$ & None & Low & More left \\
\hline 13. & None & None & None & No & None & Low & No \\
\hline 14. & None & None & None & Positive & None & Low & More right \\
\hline 15. & None & None & None & $\begin{array}{l}\text { More } \\
\text { positive }\end{array}$ & None & Low & More right \\
\hline 16. & None & None & None & Negative & None & Medium & Left \\
\hline 17. & None & None & None & No & None & Medium & No \\
\hline 18. & None & None & None & Positive & None & Medium & Right \\
\hline 19. & None & None & None & $\begin{array}{l}\text { More } \\
\text { positive }\end{array}$ & None & Medium & More right \\
\hline 20. & None & None & None & $\begin{array}{l}\text { More } \\
\text { negative }\end{array}$ & None & High & Left \\
\hline 21. & None & None & None & Negative & None & High & Left \\
\hline 22. & None & None & None & No & None & High & No \\
\hline 23. & None & None & None & Positive & None & High & Right \\
\hline 24. & None & None & None & $\begin{array}{l}\text { More } \\
\text { positive }\end{array}$ & None & High & Right \\
\hline 25. & None & None & None & $\begin{array}{l}\text { More } \\
\text { negative }\end{array}$ & None & Medium & More left \\
\hline
\end{tabular}
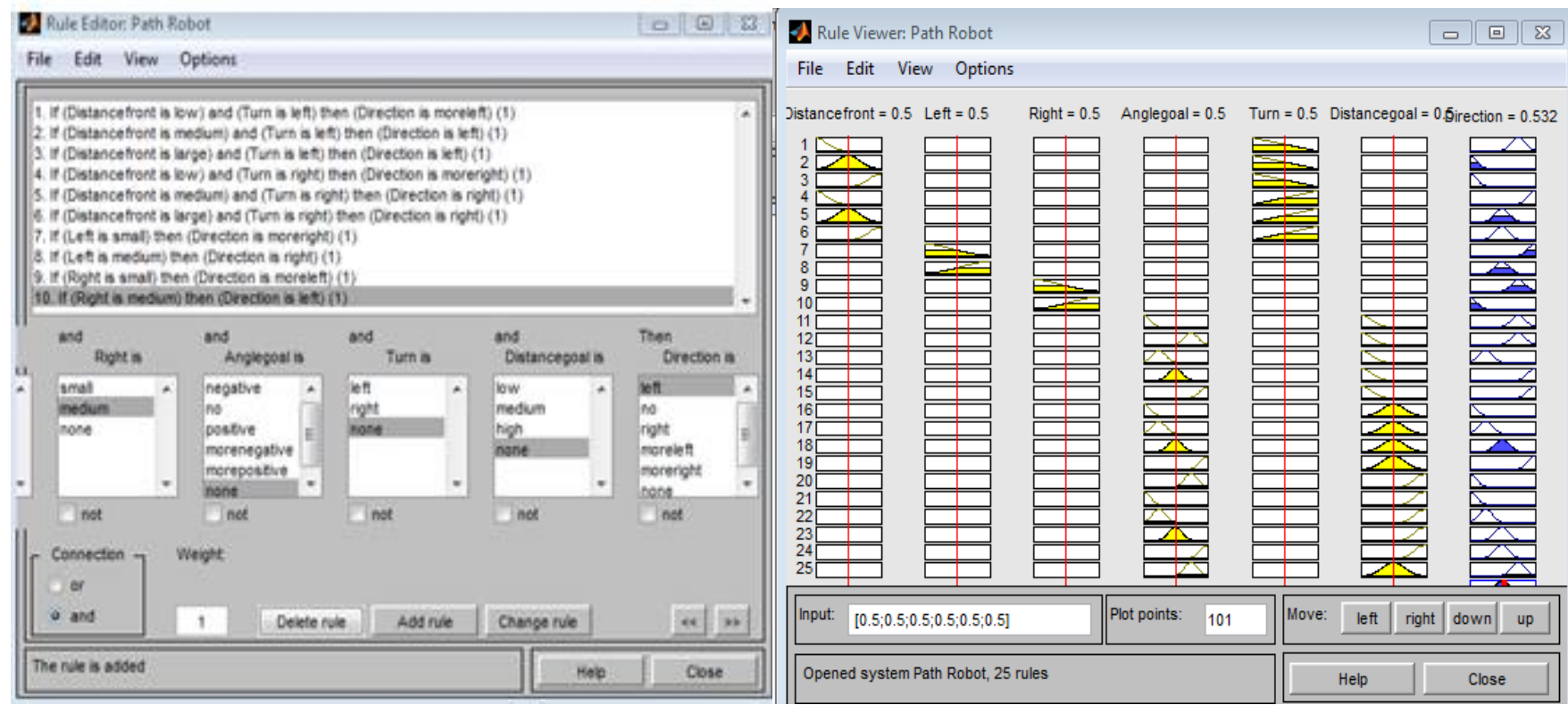

Fig 5: The rule base editor 
Published Online August 2020 in IJEAST (http://www.ijeast.com)

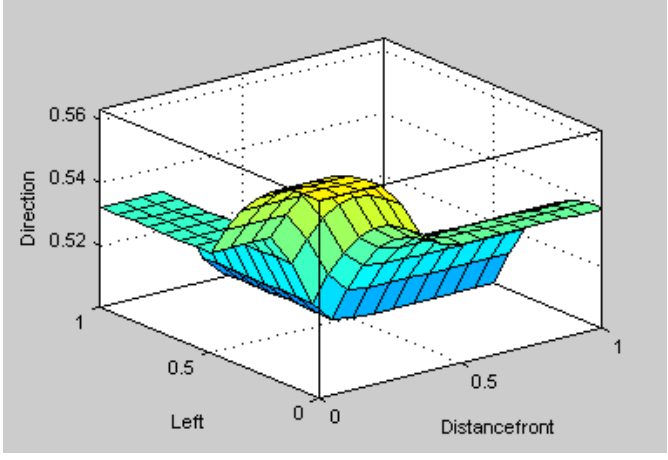

a-path 1

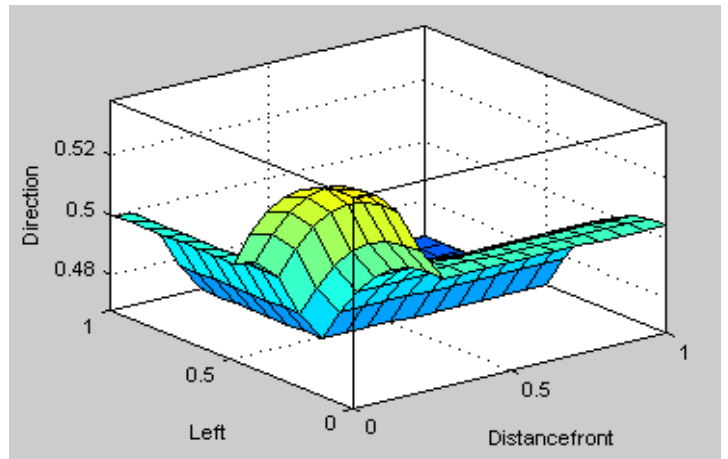

c-path 3

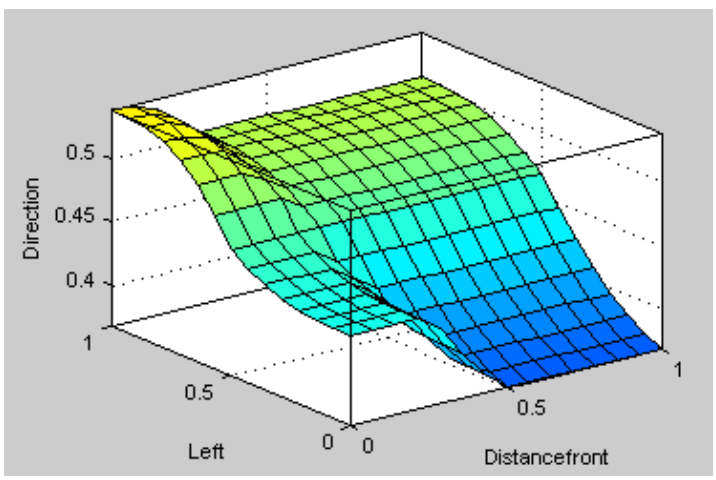

e- path 5

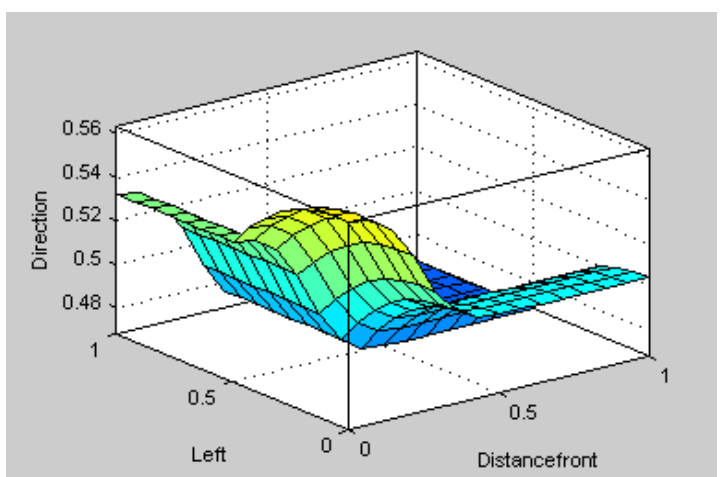

b- path 2

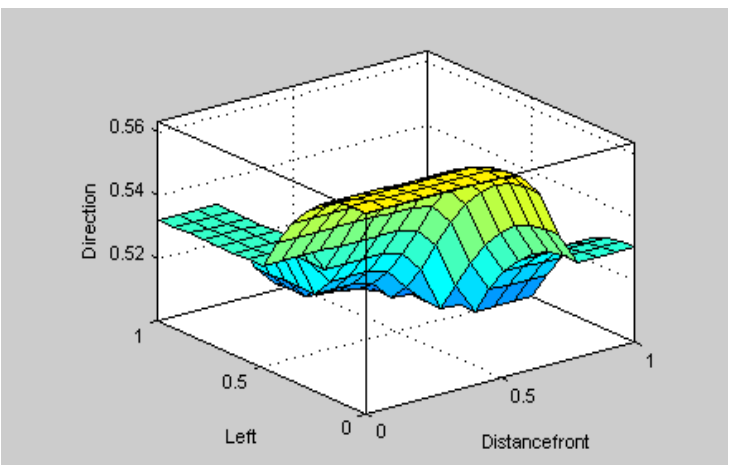

d- path 4

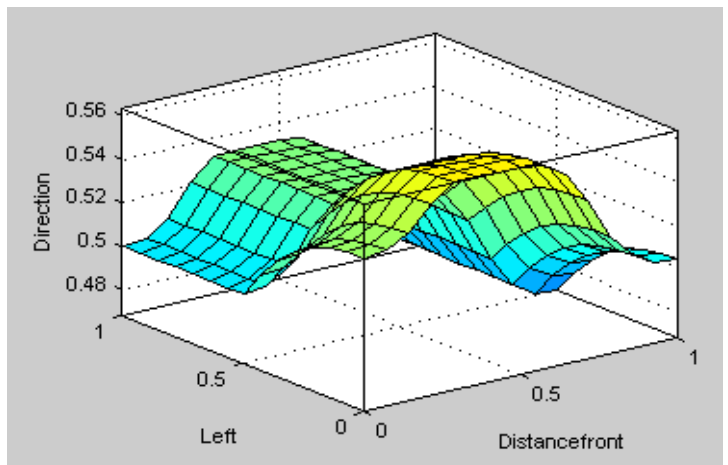

f- path 6

Fig 6: Surface viewer of all paths

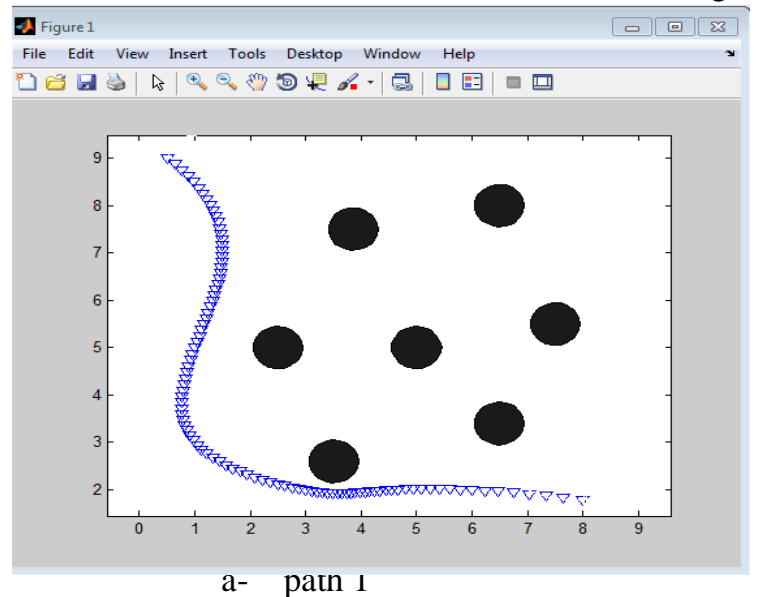

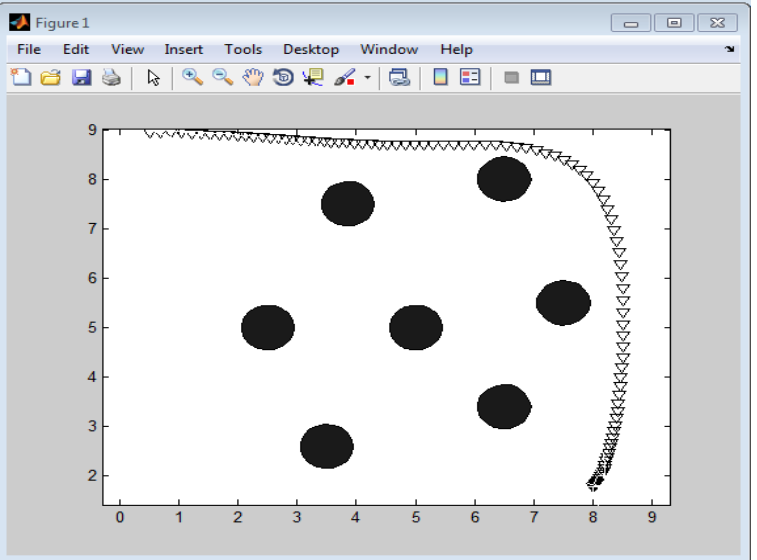

b- path 2 


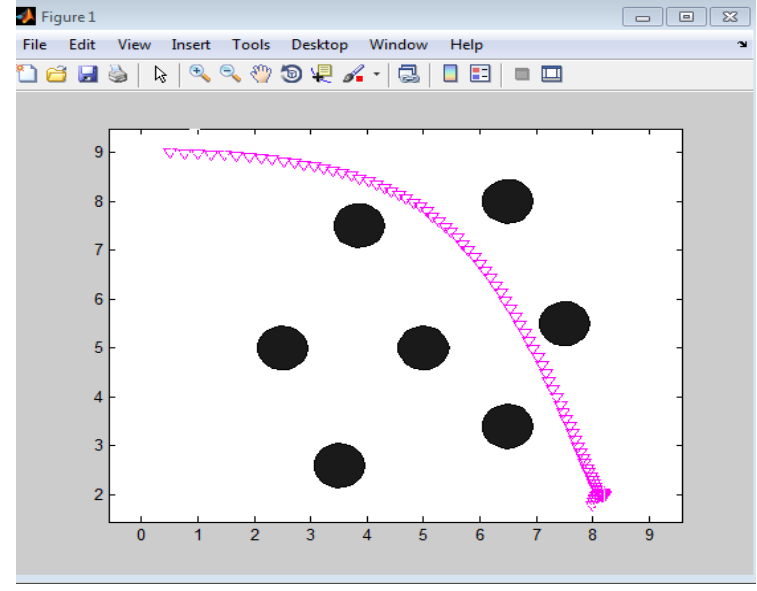

c- Path 3

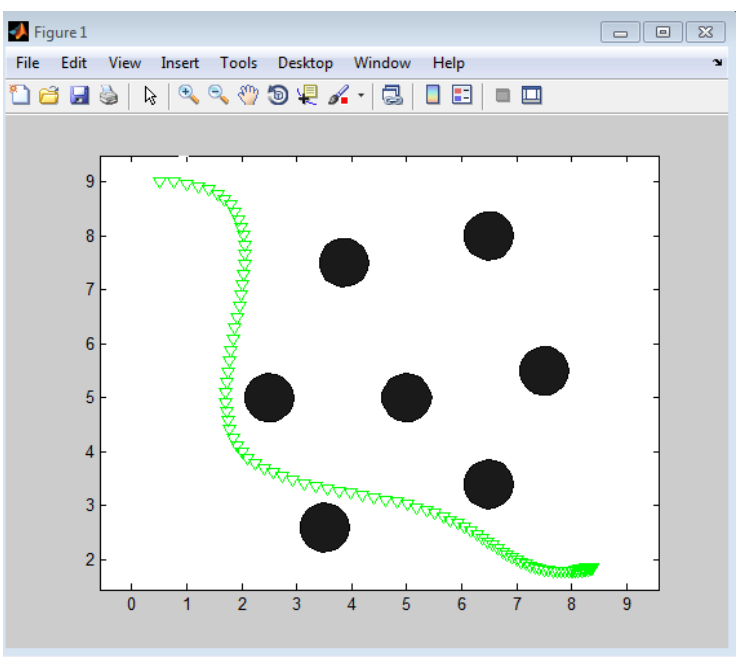

Fig 7 (a, b, c, d, e, f): Path Finding for mobile robot

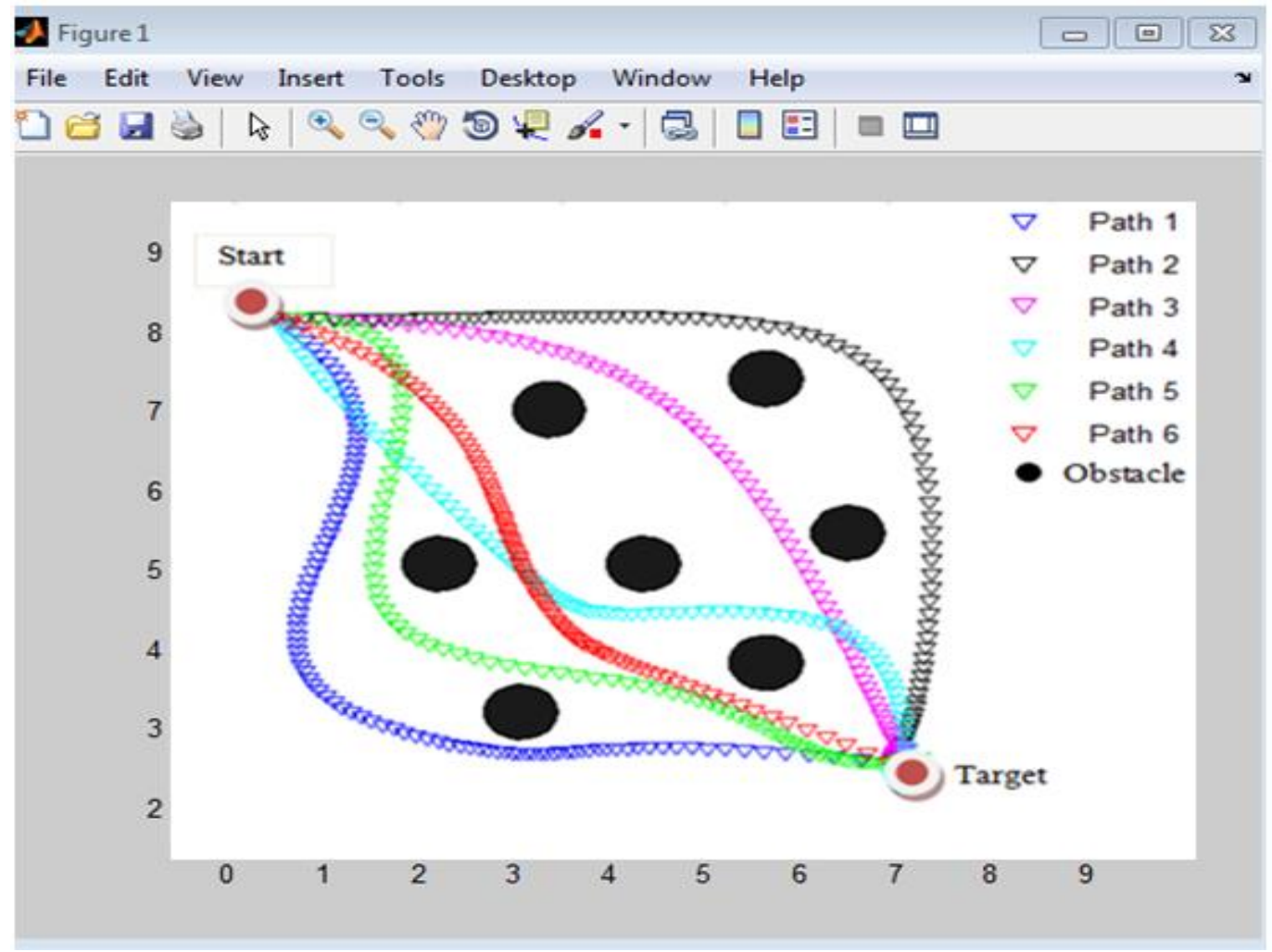

d- path

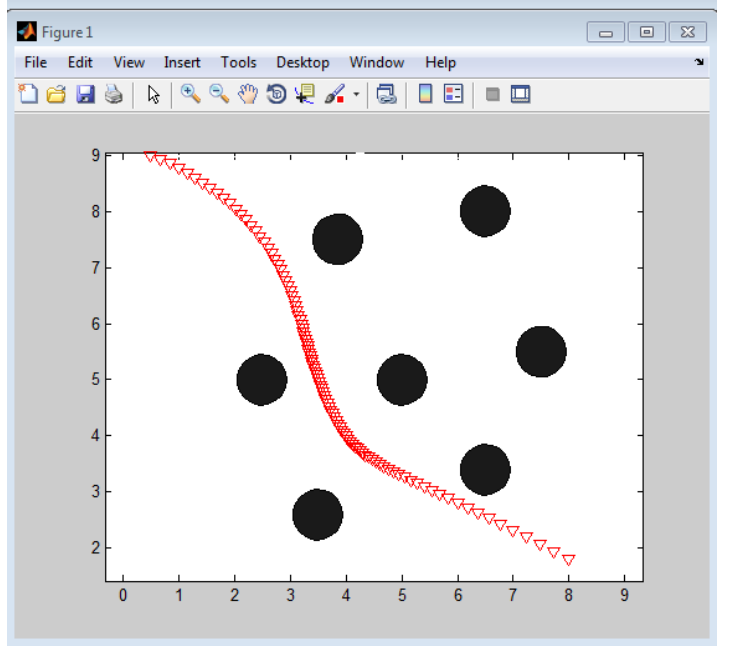

Oigure

File Edit View Insert Tools Desktop Window Help

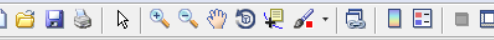

A Figure 1

File Edit View Insert Tools Desktop Window Help

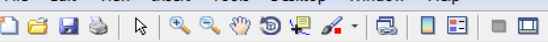

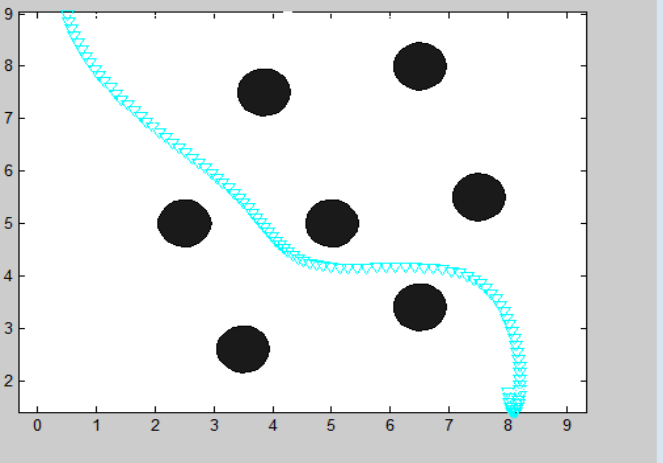


Fig 8: Multiple paths and best path (the red)

Table (II) Lengths of the paths and their implementation time

\begin{tabular}{|c|c|c|}
\hline No. of Paths & Length Path(cm) & Time Processing (sec.) \\
\hline Path 1 & 89.21 & 14.432 \\
\hline Path 2 & 88.27 & 13.211 \\
\hline Path 3 & 85.99 & 11.528 \\
\hline Path 4 & 86.38 & 11.788 \\
\hline Path 5 & 87.25 & 13.97 \\
\hline Path 6 & 85.01 & 11.31 \\
\hline
\end{tabular}

\section{CONCLUSION}

The paper discussed fuzzy logic system technology of the path and avoid the robots obstacles using image as a map. fuzzy logic is used to perform path behavior and avoidance obstacles to find the optimum path the mobile robot can access target this illustrates the success of the fuzzy logic algorithm for robots and ensure the optimum path for mobile robots which optimum path obtained from six paths, these paths takes at different rules of fuzzy logic system. which the sixth red path is best path which is characterized by its short length and the lowest processing time. Fuzzy logic technique is not an optimization technique as the particle swarm technique and the genetic algorithm are considered optimization, but in this research get several paths after changing the rules and then the optimal path is obtained from these paths in terms of short length and minimum time of implementation. This technique give the best result. As well as the sixth red path is best path which is characterized by its short length and lowest processing time, This technique applied of mobile robots

\section{REFERENCES}

[1] I. Noreen Technol, L. Pakistan , A. Khan and Z. Habib, " Optimal Path Planning for Mobile Robots Using Memory Efficient A* " IEEE International Conference on Frontiers of Information Technology (FIT) , 2017

[2] D. Huh, J. Park, U. Huh and H. Kim ," Path Planning and Navigation for Autonomus Mobile Robot" , IECON 02 IEEE annual conference, 2010.

[3] P. kumar Das, "D* lite algorithm based path planning of mobile robot in static Environment", International Journal of Computer \& Communication Technology (IJCCT), 2011.

[4] W. Khaksar and S .Vivekananthen, "A review on mobile robots motion path planning in unknown environments", IEEE International Symposium on Robotics and Intelligent Sensors (IRIS), 2015.

[5] M. Duguleana and G.Mogan, "Neural networks based reinforcement learning for mobile robots obstacle avoidance", Expert Systems with Applications , 2016.
[6] A. Adib and B. Masoumi "Mobile robots navigation in unknown environments by using fuzzy logic and learning automata", IEEE conference Artificial Intelligence and Robotics (IRANOPEN), 2017

[7] F. Nicola, Y. Fujimoto and R. Oboe "A LSTM Neural Network applied to Mobile Robots Path Planning" IEEE 16th International Conference on Industrial Informatics (INDIN) , 2018.

[8] H. Bharadwaja and V. Kumar " Comparative study of neural networks in path planning for catering robots " ELSEVIER International Conference on Robotics and Smart Manufacturing ,Procedia Computer Science, 2018.

[9] P. Sudhakara and V. Ganapathy " Trajectory Planning of a Mobile Robot using Enhanced A-Star Algorithm " Indian Journal of Science and Technology, 2016.

[10] N. Kumar, M. Takács, and Z. Vámossy, " Robot navigation in unknown environment using fuzzy logic " IEEE 15th International Symposium on Applied Machine Intelligence and Informatics (SAMI), 2017.

[11] A. Silvia Handayani, T. Dewi, N. LatifahHusni, S. Nurmaini and I. Yani, "Target tracking in mobile robot under uncertain environment using fuzzy logic controller", 4th International Conference on Electrical Engineering, Computer Science and Informatics (EECSI) IEEE, 2017.

[12] Y. CT and Cheng MF " A Study of Fuzzy Control with Ant Colony Algorithm used in Mobile Robot for Shortest Path Planning and Obstacle Avoidance " Micro-system Tech. Springer , 2018

[13] Y. Zhao, X. Chai, F. Gao, and C. Qi, " Obstacle avoidance and motion planning scheme for a hexapod robot octopus-iii ", Robotics and Autonomous Systems , 2018. 